\title{
Response Properties of Single Neurons Predicted by Sparse Representation
}

\author{
Jiqian Liu, Chengbin Zeng, and Liping Xiao
}

\begin{abstract}
Sparse representation by neuronal populations in sensory cortex has been heavily investigated and validated. Recently, it was reported that sparse representation can predict response properties of single neurons, which gives insight into the unified understanding of the responses of both neuronal populations and single neurons. The current work takes a step forward in this regard. We simulate the response properties of simple cells with a neurally plausible sparse representation model. The model turns out to explain well the response properties of single cortical neurons, such as the sharp and contrast invariant orientation tuning as well as cross orientation suppression within the classical receptive field (CRF) and surround suppression from beyond the CRF.
\end{abstract}

Index Terms-Sparse representation, orientation selectivity, cross orientation suppression, surround suppression.

\section{INTRODUCTION}

Neural mechanisms of visual information processing have been intensively investigated both at the neuronal population level and the single-neuron level for several decades. In understanding the representation of sensory stimuli by neuronal population, Barlow [1] has proposed that the goal of visual information processing is to reduce statistical redundancy in the visual signals. Olshausen and Field [2] demonstrated that an algorithm maximizing the sparseness of the code for a given image can produce basis vectors which are similar to the receptive field shapes of simple cells. Sparse representation of sensory stimuli in cortex has been widely validated [3]-[7]. On the other side, although many response properties of single neurons are well characterized, there are disagreements about the neural mechanisms underlying these observations. Ever since Hubel and Wiesel [8] first proposed a feedforward model for the orientation selectivity [9] of primary visual cortex (V1) simple cells, it has been argued whether orientation selectivity originates from excitatory convergence of lateral geniculate nucleus (LGN) afferents. This simple model predicted the tuning width of orientation selectivity should widen with increasing contrast of stimuli, which on the contrary is invariant [10], a phenomenon known as the contrast invariance of orientation selectivity. Cross

Manuscript received April 9, 2015; revised June 25, 2015. This work was supported by the Science Research Foundation for High-level Talents of Guizhou Institute of Technology XJGC20130902, and in part by the Science and Technology Foundation of Guizhou Province J[2014]2081 and by the Innovation Team of Guizhou Provincial Education Department under Grant No [2014] 34.

Jiqian Liu and Chengbin Zeng are with the School of Information Engineering, Guizhou Institute of Technology, Guiyang, 550003, P.R. China (e-mail: liujiqian@git.edu.cn).

Liping Xiao is with School of Mining Engineering, Guizhou Institute of Technology. orientation suppression in which the response of a V1 neuron to its preferred orientation is suppressed by null oriented stimuli [11], [12] has been thought to be responsible for the sharpness of orientation selectivity [13], [14]. But measurements with intracellular recordings [15], [16] indicated that such kind of inhibition could not be involved in cross orientation suppression. Another phenomenon known as surround suppression, that is, the presence of stimuli in the non-classical receptive field (nCRF) of neurons can suppress their spiking responses [17]-[19], has attracted much interest in the last decade. Differently from cross orientation suppression, surround suppression is strongest when the stimulus in the nCRF has a parallel orientation (iso-orientation) and exhibits contrast dependent size tuning [20]. The spatial extent seems not to support the suppression induced by far surround stimuli [21]. The lack of global information in the observations made in a very limited area could be the main reason for these disagreements.

In this paper, we simulate the response properties of simple cells with a sparse representation algorithm, demonstrating that the conflicting measurement results of single neurons may occur as a consequence of the neuronal populations performing sparse representation. Recently, some other studies have reported that several classical receptive field (CRF) and non-classical receptive field (nCRF) response properties of single neurons are well simulated by sparse representation [22]-[24]. To further testify this hypothesis, we use a neurally plausible sparse representation model to simulate the emergence of several known simple cell response properties. For a unified understanding of the responses of both neuronal populations and single neurons in early visual cortex, our work takes it a step forward.

\section{THE $L_{0}$ NORM BASED SPARSE REPRESENTATION}

In the model presented by Olshausen and Field [2], there were only two global objectives to be optimized: that the representation is sparse and that the representation error is small. Rehn and Sommer [25] then introduced the $L_{0}$ norm to replace the smoothness prior to force the representation to be sparse. The sparse representation problem modeled with a $L_{0}$ norm term is given by

$$
\min \left\{\left(x-\sum_{j} a_{j} w_{j}\right)^{2}+\lambda\|a\|_{0}\right\}
$$

where $\boldsymbol{x}$ is the input signal, $w_{j}$ is the basis vector corresponding to the receptive field of an active neuron, $a_{j}$ is the coefficient of $w_{j}, a$ is a vector whose $j$ th component is 
$a_{j}$, and $\lambda$ is the trade-off parameter.

A large number of methods have been proposed for this problem. Iterative thresholding algorithms (ITA) solve sparse representation in an iterative framework using thresholding [26]-[28]. In these methods, the input is approximated iteratively while the representation is made sparser by a thresholding mechanism at each iteration step. It is widely believed that lateral inhibition play an important role for the implementation of sparse representation in visual cortex [29]. The lateral inhibitory coefficients are consistent with those obtained from physiological data [30]. In this paper, a neurally plausible sparse representation model with lateral inhibition and thresholding is adopted. Furthermore, non-negative data processing is performed. With these modifications, the laterally inhibited non-negative sparse representation algorithm (LINSR) is proposed in Table I.

TABLE I: LATERALLY INHIBITED NON-NEGATIVE SPARSE REPRESENTATION

INPUT: the signal $x$ and the set of basis vectors $W$.

OUTPUT: the approximation $\hat{x}$ of $x$.

Initially, compute $y_{i}^{(0)}=Y_{i}=x \cdot w_{i}$ for all $w_{i} \in W$.

Repeat the following two steps several times for all $i$ :

Step 1. If $y_{i}^{(\tau)}<0$, set $y_{i}^{(\tau)}=0$.

Step 2. Calculate $y_{i}^{(\tau+1)}=Y_{i}-\sum_{k \neq i} C_{k, i} y_{k}^{(\tau)}$ where $C_{k, i}$ is also set to be 0 if it is smaller than a given threshold $\mu>0$.

In Table I, $C_{k, i}$ is the inhibitory coefficient of the $k$ th output neuron on the $i$ th output neuron which is calculated by $C_{k, i}=\frac{w_{k} w_{i}}{\left\|w_{k}\right\|^{2}}$ [30]. In the implementation process of LINSR, a large initial positive response may be reduced to zero. Correspondingly, some active output neurons will be forced to be inactive by lateral inhibition resulting that only a smaller number of output neurons left for the representation of $\boldsymbol{x}$. It means that the representation is forced to be sparser and more accurate, and thus the two global objectives of the sparse representation model are achieved.

\section{EXPERIMENTAL RESULTS}

\section{A. Sparse Representation of Image Patches}

We test the LINSR algorithm with a $250 \times 250$ sized "Lena" image (see Fig. 1(a)). To make the test simpler, we use a group of two dimensional trigonometric functions and two Gaussian functions illustrated in Fig. 1(b) as the basis set. The two dimensional trigonometric functions are given by

$$
u(x, y)=\sin \left(\alpha \pi f(y \cos i \Delta \theta-x \sin i \Delta \theta)+\frac{k \pi}{2}\right)
$$

where $\alpha$ is a scalar, $f=1+0.7 n$, and $\Delta \theta=\pi / 6$. Both the spatial frequency $f$ and the value of $\Delta \theta$ are carefully selected to generate sinusoidal gratings that are slightly correlated. By doing this, the generated basis functions are neither highly correlated to avoid the redundancy in the representation nor too dispersed to avoid the loss important information in the input. In the visual cortex, the value of $\Delta \theta$ may be a little smaller because the Gabor-like receptive fields of V1 neurons are more localized than the sinusoidal functions.

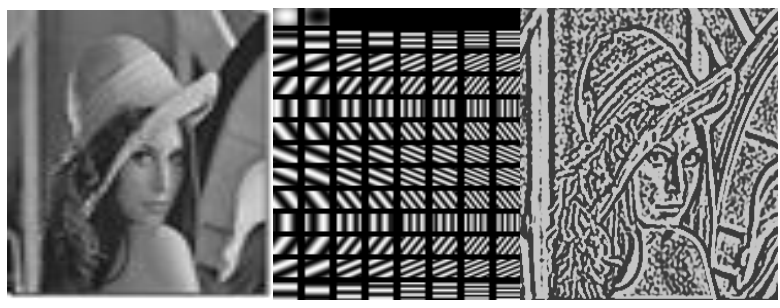

(a) (b) (c)

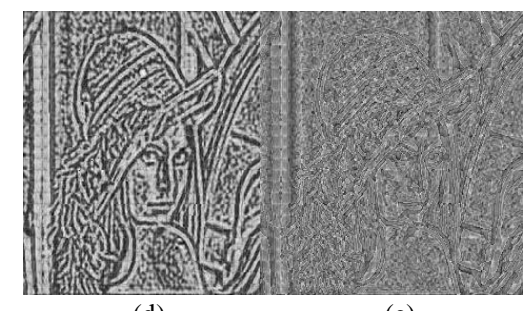

(d)

(e)

Fig. 1. Illustration of the DoG filtered "Lena" image being reconstructed by the LINSR algorithm. (a) The "Lena" image with a size of $250 \times 250$ pixels. (b) The filters used in this paper. (c) The DoG filtered "Lena" image which is cut into $25 \times 25$ patches with a size of $10 \times 10$ pixels as the inputs of the LINSR algorithm. (d) The stitched image from the reconstructed patches. (e) The error image between the DoG filtered "Lena" and reconstructed result.

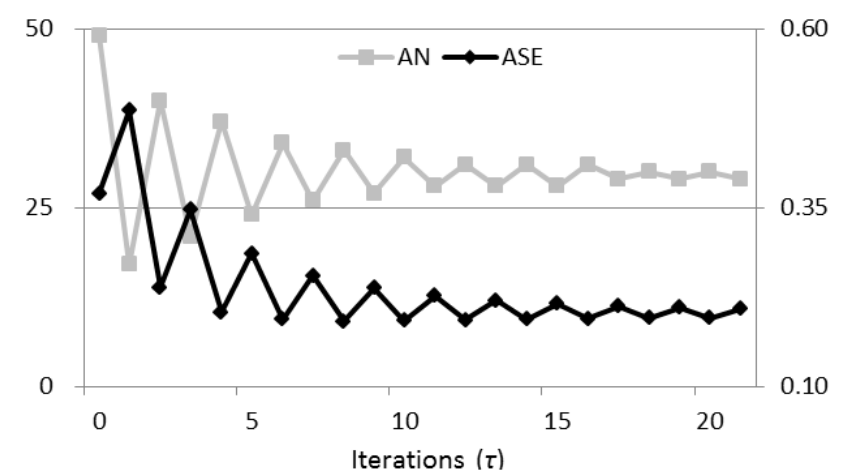

Fig. 2. Trend of the average number (AN) of selected basis functions and the average squared error (ASE) in the first iterations of the reconstruction process for the "Lena" image.

As mentioned above, the "Lena" image will be filtered with DoG at first. Then the DoG filtered "Lena" image (see Fig. $1(\mathrm{c}))$ is cut into $25 \times 25$ patches with a size of $10 \times 10$ pixels as the input data set. Each of the image patches, corresponding to $\boldsymbol{x}$, is reconstructed by the LINSR algorithm one by one. At last, we stitch all the reconstructed image patches, corresponding to $\hat{x}$, back together into the new image as shown in Fig. 1(d). Comparing Fig. 1(c) with Fig. 1(d), we see that the DoG filtered image is reconstructed very well. The error image is given in Fig. 1(e).

We provide an error analysis of the first iterations of the LINSR algorithm, and the result is shown in Fig. 2 where the average number of the basis functions selected for the reconstruction of each image patch is also plotted. When to calculate the average squared error, all image patches are normalized to remove amplitude dependence. From Fig. 2 we can see that the iterative procedure tends to converge after about 15 iterations. This demonstrates the time course of reconstruction can be very short. 




(a)

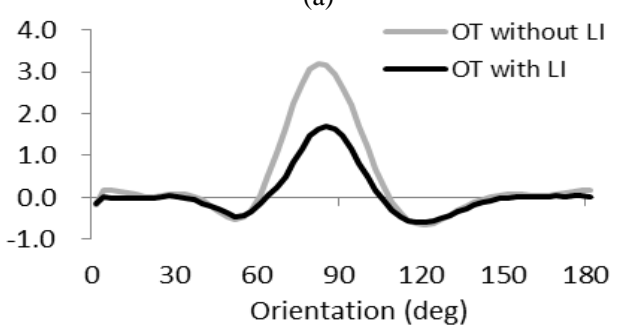

(b)

Fig. 3. Lateral inhibition sharpens orientation tuning (OT). The orientation tuning curves calculated with lateral inhibition are sharper than those without lateral inhibition. (a) The grating stimuli are not filtered before fed to the input of the network. (b) The grating stimuli are filtered.

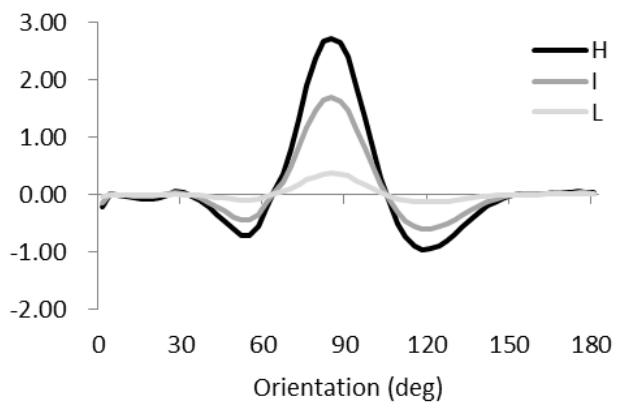

Fig. 4. Illustration of the contrast invariance of orientation tuning. The tuning width remains unchanged for stimuli at high $(\mathrm{H})$, intermediate (I) and low (L) contrasts.

\section{B. Neuronal Response Properties}

We analyze the response properties of single neurons with sinusoidal gratings over the full range of orientation angles. The gratings are generated in the same way as the basis vectors to make sure they can elicit a maximum response of the output neurons as those used in physiological experiments. The simulation result shown in Fig. 3 indicates that the selected output neuron exhibits sharp orientation tuning (OT), and the tuning curve calculated only with the LGN inputs can be much broader than that with lateral inhibition. These results are consistent with the observations that orientation selectivity results mainly from broader tuning of excitation and can be reduced by blocking cortical inhibition [31], [32]. The contrast invariance of orientation selectivity is also predicted by our model (see Fig. 4). The tuning width, an interval where the neuron shows a positive response, remains unchanged with respect to contrast. The Mexican-hat appearance of some tuning curves as illustrated in Fig. 5 has been reported and modeled by Ringach et al. [33], [34].

As suggested by MacEvoy et al. [35], we use the population response properties to examine cross orientation suppression. Responses of output neurons preferring the same orientation angle are averaged. The average population response profiles are plotted in Fig. 6 where the profile obtained at low contrast for superimposed gratings maintain the same structure as those at high contrasts. This is an important physiological observation [35] against the prediction of a purely feedforward model without lateral inhibition [36].

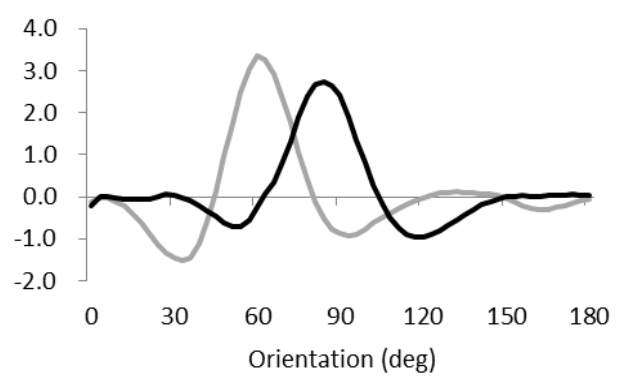

Fig. 5. Simulated orientation tuning curves of two output neurons, both of which have a Mexican-hat shape with little difference.

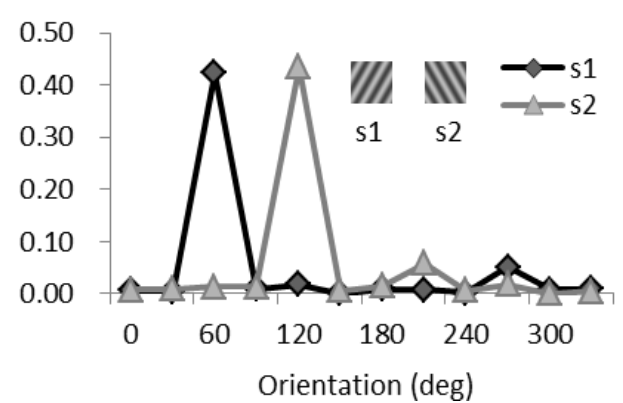

(a)

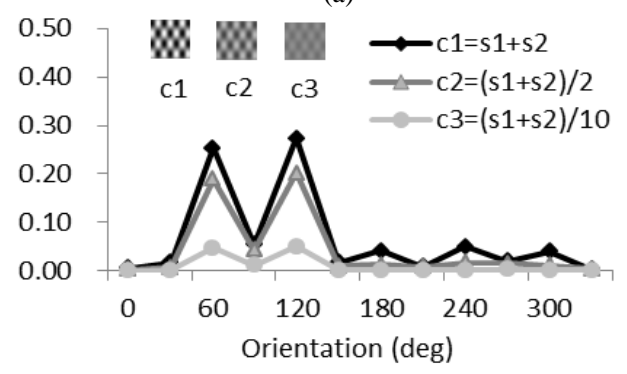

(b)



(c)



(d)

Fig. 6. Average responses of output neurons preferring the same orientation to single and superimposed gratings. Each data point represents the average response of output neurons that prefer the same orientation angle. $(a, c)$ Average population response profiles for two single gratings s1 and s2. (b, d) Average population response profiles for superimposed gratings c1, c2 and c3 at different contrasts. 


\section{CONCLUSION}

In this paper, we demonstrate that most response properties of simple cells may occur as a result of neuronal populations performing sparse representation in V1. To achieve this purpose, the neurally plausible sparse representation model named LINSR is proposed. The sharp and contrast invariant orientation selectivity, cross orientation suppression and surround suppression are well simulated by LINSR. Future work will focus on the development of more neurally plausible sparse representation models and the simulation of more complex response properties of single neurons.

\section{REFERENCES}

[1] H. B. Barlow, "Possible principles underlying the transformation of sensory messages," Sensory Communication, pp. 217-234, 1961.

[2] B. A. Olshausen and D. J. Field, "Emergence of simple-cell receptive field properties by learning a sparse code for natural images," Nature, vol. 381, pp. 607-609, 1996.

[3] W. E. Vinje and J. L. Gallant, "Sparse coding and decorrelation in primary visual cortex during natural vision," Science, vol. 287 , no 5456, pp. 1273-1276, 2000.

[4] J. Perez-Orive, O. Mazor, G. C. Turner, S. Cassenaer, R. L. Wilson, and G. Laurent, "Oscillations and sparsening of odor representations in the mushroom body," Science, vol. 297, no. 5580, pp. 359-365, 2002.

[5] M. S. Lewicki, "Efficient coding of natural sounds," Nature Neuroscience, vol. 5, pp. 356-363, 2002.

[6] G. Laurent, "Olfactory network dynamics and the coding of multidimensional signals," Nature Reviews Neuroscience, vol. 3, pp. 884-895, 2002.

[7] E. C. Smith and M. S. Lewicki, "Efficient auditory coding," Nature, vol. 439, pp. 978-982, 2006.

[8] D. H. Hubel and T. N. Wiesel, "Receptive fields, binocular interaction and functional architecture in the cat's visual cortex," The Journal of Physiology, vol. 160, pp. 106-154, 1962.

[9] D. H. Hubel and T. N. Wiesel, "Receptive fields of single neurones in the cat's striate cortex," The Journal of Physiology, vol. 148, pp. 574-591, 1959

[10] B. C. Skottun, A. Bradley, G. Sclar, I. Ohzawa, and R. D. Freeman, "The effects of contrast on visual orientation and spatial frequency discrimination: a comparison of single cells and behavior," Journal of Neurophysiology, vol. 57, no. 3, pp. 773-786, 1987.

[11] M. C. Morrone, D. C. Burr, and L. Maffei, "Functional implications of cross-orientation inhibition of cortical visual cells. I. neurophysiological evidence," Proceedings of the Royal Society of London Series B Biological Sciences, vol. 216, pp. 335-354, 1982.

[12] G. C. DeAngelis, J. G. Robson, I. Ohzawa, and R. D. Freeman, "Organization of suppression in receptive fields of neurons in cat visual cortex," Journal of Neurophysiology, vol. 68, pp. 144-163, 1992.

[13] H. Sompolinsky and R. Shapley, "New perspectives on the mechanisms for orientation selectivity," Current Opinion in Neurobiology, vol. 7, pp. 514-522, 1997.

[14] T. Z. Lauritzen and K. D. Miller, "Different roles for simple-cell and complex-cell inhibition in V1," The Journal of Neuroscience, vol. 23 , pp. 10201-10213, 2003.

[15] J. S. Anderson, M. Carandini, and D. Ferster, "Orientation tuning of input conductance, excitation, and inhibition in cat primary visual cortex," Journal of Neurophysiology, vol. 84, no. 2, pp. 909-926, 2000.

[16] N. J. Priebe and D. Ferster, "Mechanisms underlying cross-orientation suppression in cat visual cortex," Nature Neuroscience, vol. 9, pp. 552-561, 2006.

[17] C. Blakemore and E. A. Tobin, "Lateral inhibition between orientation detectors in the cat's visual cortex," Experimental Brain Research, vol. 15, no. 4, pp. 439-440, 1972.

[18] L. Maffei and A. Fiorentini, "The unresponsive regions of visual cortical receptive fields," Vision Research, vol. 16, no. 10, pp. 1131-1139, 1976.

[19] J. Allman, F. Miezin, and E. McGuinness, "Stimulus specific responses from beyond the classical receptive field: Neurophysiological mechanisms for local-global comparisons in visual neurons," Annual Review of Neuroscience, vol. 8, pp. 407-430, 1985 .
[20] M. P. Sceniak, D. L. Ringach, M. J. Hawken, and R. Shapley, "Contrast's effect on spatial summation by macaque V1 neurons," Nature Neuroscience, vol. 2, pp. 733-739, 1999.

[21] A. Angelucci, J. B. Levitt, E. J. S. Walton, J.-M. Hupe, J. Bullier, and J. S. Lund, "Circuits for local and global signal integration in primary visual cortex," the Journal of Neuroscience, vol. 22, pp. 8633-8646, 2002.

[22] J. J. Hunt, P. Dayan, and G. J. Goodhill, "Sparse coding can predict primary visual cortex receptive field changes induced by abnormal visual input," PLoS Computational Biology, vol. 9, 2013.

[23] A. D. Giorno, M. Zhu, and C. J. Rozell, "A sparse coding model of V1 produces surround suppression effects in response to natural scenes," BMC Neuroscience, vol. 14, p. 335, 2013.

[24] M. Zhu and C. J. Rozell, "Visual nonclassical receptive field effects emerge from sparse coding in a dynamical system," PLoS Computational Biology, vol. 9, 2013.

[25] M. Rehn and F. T. Sommer, "A network that uses few active neurones to code visual input predicts the diverse shapes of cortical receptive fields," Journal of Computational Neuroscience, vol. 22, pp.135-146, 2007.

[26] K. K. Herrity, A. C. Gilbert, and J. A. Tropp, "Sparse approximation via iterative thresholding," in Proc. IEEE International Conference on Acoustics, Speech and Signal Processing, 2006, vol. 3.

[27] M. Fornasier and H. Rauhut, "Iterative thresholding algorithms," Applied and Computational Harmonic Analysis, vol. 25, pp. 187-208, 2008.

[28] T. Blumensath and M. E. Davies, "Iterative thresholding for sparse approximations," Journal of Fourier Analysis and Applications, vol. 14, pp. 629-654, 2008

[29] B. A. Olshausen, "Principles of image representation in visual cortex," The Visual Neurosciences, pp. 1603-1615, 2003.

[30] J. Liu and Y. Jia, "A lateral inhibitory spiking neural network for sparse representation in visual cortex," Advances in Brain Inspired Cognitive Systems, vol. 7366, pp. 259-267, 2012.

[31] A. M. Sillito, "The contribution of inhibitory mechanisms to the receptive field properties of neurones in the striate cortex of the cat," The Journal of Physiology, vol. 250, pp. 305-329, 1975.

[32] A. M. Sillito, J. A. Kemp, J. A. Milson, and N. Berardi, "A re-evaluation of the mechanisms underlying simple cell orientation selectivity," Brain Research, vol. 194, no. 2, pp. 517-520, 1980.

[33] D. L. Ringac, M. J. Hawken, and R. Shapley, "Dynamics of orientation tuning in macaque primary visual cortex," Nature, vol. 387, pp 281-284, 1997.

[34] D. L. Ringach, M. J. Hawken, and R. Shapley, "Dynamics of orientation tuning in macaque V1: The role of global and tuned suppression," Journal of Neurophysiology, vol. 90, no. 1, pp. 342-352, 2003.

[35] S. P. MacEvoy, T. R. Tucker, and D. Fitzpatrick, "A precise form of divisive suppression supports population coding in the primary visual cortex," Nature Neuroscience, vol. 12, pp. 637-645, 2009.

[36] I. M. Finn, N. J. Priebe, and D. Ferster, "The emergence of contrast-invariant orientation tuning in simple cells of cat visual cortex," Neuron, vol. 54, no. 1, pp. 137-152, 2007.

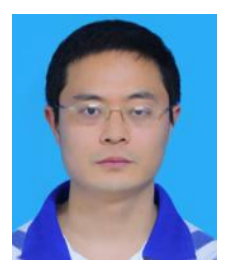

Jiqian Liu was born in 1979 in Shandong Province of China. He obtained a Ph.D degree in computer science and technology from the Beijing Institute of Technology in 2013, and received the bachelor degree from Shandong University of Science and Technology in 2001. His research interests include neural network, computational neuroscience, computer vision and pattern recognization.

Dr. Liu is an associate professor of School of Information Engineering, Guizhou Institute of Technology now. He worked as a teaching assistant at School of Science, Hebei University of Engineering from 2001 to 2005.

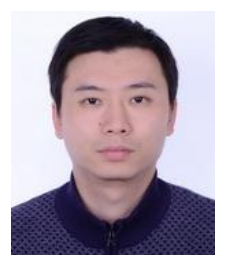

Chengbin Zeng was born in 1979 in Guizhou Province of China. He obtained his Ph.D degree in computer science and technology from Beijing University of Posts and Telecomm in 2011, and received the bachelor degree and master degree both from Guizhou University in 2001 and 2006. His research interests include computer vision, deep learning.

Dr. Zeng is an associate professor of School of Information Engineering, Guizhou Insitute of Technology now. 
Liping Xiao is an associate professor of School of Mining Engineering, Guizhou Institute of Technology. She received the bachelor degree in 1991 from the Department of Mining, Guizhou University of Technology, and received the master degree in 2005 in software engineering from Wuhan University. From October 2009 to October 2010, Xiao was a visiting scholar at Germany In Went. Her primary research interest lies in the area of the computer science and application.

Ms. Xiao is an associate professor at School of Mining Engineering, Guizhou Institute of Technology up to now. From Nov. 2008 to Dec. 2013 she works as an associate professor at the vocational and technical college of Guizhou University. From Jan. 2003 to Oct. 2008, she is a senior lecturer at School of Construction Management, Guizhou University of Technology. 